\title{
Correction to: Prenatal Ultrasonic Diagnosis and Prognostic Analysis of Isolated Left Subclavian Artery (ILSCA) and Left Brachiocephalic Trunk (ILBCT) Malformation
}

Yuntao $\mathrm{Li}^{1} \cdot$ Xiaohong Zhang ${ }^{2} \cdot$ Yani Yan $^{2} \cdot$ Qiuyan $\mathrm{Pei}^{2}$

Published online: 12 October 2018

๑) Springer Science+Business Media, LLC, part of Springer Nature 2018

\section{Correction to: Pediatric Cardiology https://doi.org/10.1007/s00246-018-1945-1}

The article Prenatal Ultrasonic Diagnosis and Prognostic Analysis of Isolated Left Subclavian Artery (ILSCA) and Left Brachiocephalic Trunk (ILBCT)Malformation, written by Yuntao Li, Xiaohong Zhang, Yani Yan and Qiuyan Pei, was originally published electronically on the publisher's internet portal (currently SpringerLink) on August 2018 with open access.
The original article can be found online at https://doi.org/10.1007/ s00246-018-1945-1.

Qiuyan Pei

pqypei@126.com

1 Department of Obstetric Ultrasonography, Peking University People' Hospital, No. 11 of Nanda Street, Xicheng District, Beijing 100044, China

2 Department of Obstetric, Peking University People' Hospital, Beijing 100044, China
With the author(s)' decision to step back from Open Choice, the copyright of the article changed on October 2018 to $\odot$ Springer Science + Business Media, LLC, part of Springer Nature 2018 and the article is forthwith distributed under the terms of copyright. 